\title{
Brain MR Imaging Findings of Cardiac-Type Fabry Disease with an IVS4+919G > A Mutation
}

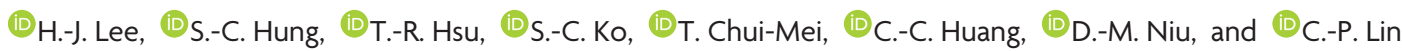

\begin{abstract}
BACKGROUND AND PURPOSE: A high incidence of cardiac-type Fabry disease with an $\alpha$-galactosidase A mutation, IVS4 + 919 G $>$ A, has been identified in the Taiwanese population. The neurologic manifestation has not been understood in this specific cardiac variant. This study aimed to investigate the typical imaging features of classic Fabry disease in patients with IVS4 Fabry disease.
\end{abstract}

MATERIALS AND METHODS: Twenty-six patients with IVS4-type Fabry disease (20 men and 6 women; age range, 43-71 years; median age, 61 years) and 26 age- and sex-matched healthy controls (age range, 44-68 years; median age, 60 years) were analyzed for white matter hyperintensities, the pulvinar sign, and basilar artery diameter. The volumes of white matter hyperintensities were calculated by comparison with an in-house data base of 276 controls.

RESULTS: Infarctions were found in 9 patients with IVS4 Fabry disease (35\%) and in none of the healthy controls $(P=.001)$. A pulvinar sign was found in 8 patients with IVS4 Fabry disease $(30 \%)$ and in none of the healthy controls $(P=.002)$. No significant difference was found in Fazekas scale scores for white matter hyperintensities; however, white matter hyperintensity volume in the deep white matter was higher in patients with IVS4 Fabry disease than in those from the healthy control data base $(P=.004)$.

CONCLUSIONS: Along with its involvement of the cardiac system, IVS4-type Fabry disease has features similar to those of classic Fabry disease and a higher frequency of deep white matter hyperintensities and a higher incidence of infarctions and pulvinar signs than in healthy controls.

ABBREVIATIONS: $\mathrm{BA}=$ basilar artery; $\mathrm{GLA}=\alpha$-galactosidase $\mathrm{A} ; \mathrm{WMH}=$ white matter hyperintensity

C lassic Fabry disease is a multisystem X-linked lysosomal disorder due to lysosomal $\alpha$-galactosidase A (GLA) deficiency, which subsequently leads to accumulation of glycosphingolipids, primarily globotriaosylceramide, throughout the body. ${ }^{1}$ The disease results in severe renal, cardiac, and central nervous system complications in adulthood. On brain MR imaging, classic Fabry disease is characterized by white matter hyperintensities, infarcts, and dolichoectasia. ${ }^{2}$

In the general population, the incidence of Fabry disease has

Received August 27, 2015; accepted after revision November 29.

From the Departments of Radiology (H.-J.L., S.-C.H., T.C.-M.) and Pediatrics (T.-R.H., D.-M.N.) and Taiwan Health-Tech Imaging Center (S.-C.K.), Taipei Veterans General Hospital, Taipei, Taiwan; and School of Medicine (H.-J.L., S.-C.H., T.-R.H., S.-C.K., T.C.-M., C.-C.H., D.-M.N., C.-P.L.) and Department of Biomedical Imaging and Radiological Sciences (S.-C.H., C.-C.H., C.-P.L.), National Yang-Ming University, Taipei, Taiwan.

H.-J. Lee and S.-C. Hung are co-first authors.

Please address correspondence to Ching-Po Lin, PhD, Department of Biomedical Imaging and Radiological Sciences, National Yang-Ming University, 155, Li-Nong St Section 2, Peitou, Taipei, Taiwan, 112, Republic of China; e-mail: cplin@ym.edu.tw; and Dau-Ming Niu, MD, Department of Pediatrics, Taipei Veterans General Hospital, 201 Shih-Pai Rd, Section 2, Peitou, Taipei, Taiwan, 112, Republic of China; e-mail: dmniul111@yahoo.com.tw

http://dx.doi.org/10.3174/ajnr.A4677 been reported as 1 in 40,000-117,000 live births. However, our previous studies by using neonate screening had identified a high incidence (approximately 1 in 1600 males) of a cardiac variant resulting from a GLA mutation, IVS4 + 919G >A (IVS4-type). ${ }^{3,4}$ Another study revealed that 12 of 10,499 males (1/875) and 24 of the 9564 females $(1 / 399)$ had the IVS4 +919 G $>$ A mutation in neonate screening. ${ }^{5}$ The natural course of the IVS4-type Fabry disease is still largely unknown. The intronic mutation (IVS4 + 919G $>$ A) was reported to be a "cardiac-type" Fabry mutation, ${ }^{6}$ which may present with asymptomatic, mild symptomatic as microalbuminuria and retinal vessel tortuosity, to severe cardiac symptoms causing significant morbidity after the fifth decade of life. However, the neurologic symptoms in the specific subtype have never been understood. Therefore, the current study aimed to analyze the degrees of CNS involvement in IVS4-type Fabry disease by retrospectively comparing brain imaging results of this patient population with images from a healthy control data base.

\section{MATERIALS AND METHODS Population}

This study was approved by the institutional review board at our institution. In total, 920 patients (498 men and 422 women) were 

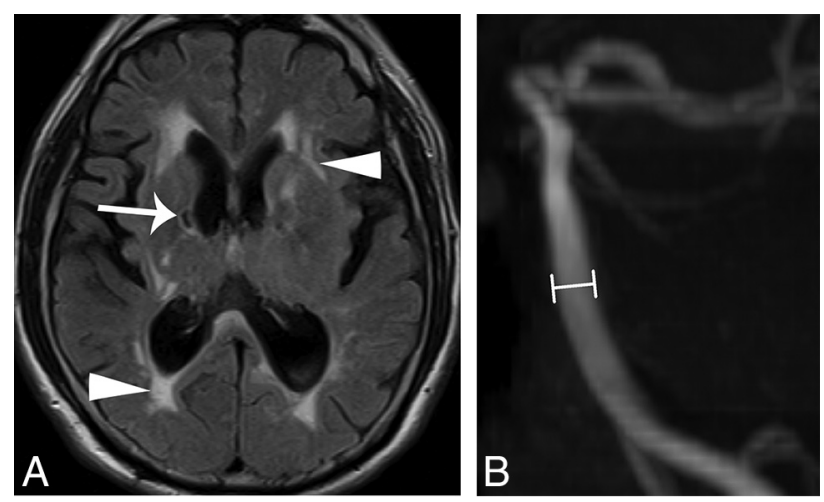

FIG 1. A 54-year-old man with IVS4-type Fabry disease had hypertrophic cardiomyopathy, headache, and stroke. Brain MR $(A)$ axial T2 FLAIR image reveals old lacunar infarcts at the bilateral internal capsules (arrow) and increased deep white matter hyperintensities (arrowheads). $B$, The diameter at the middle segment of the basilar artery (lines) was measured as $3.2 \mathrm{~mm}$ on a 3D time-of-flight MR angiogram.

diagnosed with Fabry disease with IVS4 + 919G $>$ A mutation. Twenty-six patients (20 men and 6 women; age range, 43-71 years; median age, 61 years) who had brain MR imaging examinations from 2010 to 2014 were enrolled in the study. The MR images were retrospectively reviewed by 2 certified neuroradiologists (S.-C.H. and H.-J.L.) by consensus and were compared with those of 26 sex- and age-matched healthy controls (20 men and 6 women; age range, $44-68$ years; median age, 60 years). The age of healthy controls was matched to the patient age \pm 2 years. The MR imaging findings in the sexes between IVS4-type of Fabry disease and age-matched healthy controls were also compared to understand the sex-based differences. For the healthy control groups, we excluded individuals with abnormal renal function and abnormal electrocardiogram findings to lower the possibility of undiagnosed Fabry disease, and we excluded patients with bilateral fetal origin of the posterior cerebral artery to avoid the normal variation of a small-sized basilar artery (BA).

The volumes of white matter hyperintensities of patients with Fabry disease were also quantitatively calculated and compared with another in-house data base of 276 healthy controls.

\section{Clinical Assessment}

With the exception of 1 patient who was lost to follow-up, a questionnaire of neurologic and neuropsychiatric symptoms was administered to the 25 patients. The questionnaire evaluated symptoms of stroke, sudden onset of numbness or weakness in the extremities, asymmetric facial expression, dysarthria, sudden onset of blurred vision or diplopia, depression, personality changes, forgetfulness, tinnitus, and vertigo.

\section{Qualitative and Semi-Quantitative Evaluation}

The presence and location of an infarction was categorized into the following groups: none, anterior circulation alone, posterior circulation alone, and both anterior and posterior circulations (Fig 1A). High signal changes on T1-weighted images at the bilateral lateral pulvinar were considered pulvinar signs (Fig 2). ${ }^{7}$

Deep white matter hyperintensities (WMHs) on T2-weighted images were semi-quantitatively graded by using the Fazekas scale, ${ }^{8}$ in which WMHs are classified by the following scoring

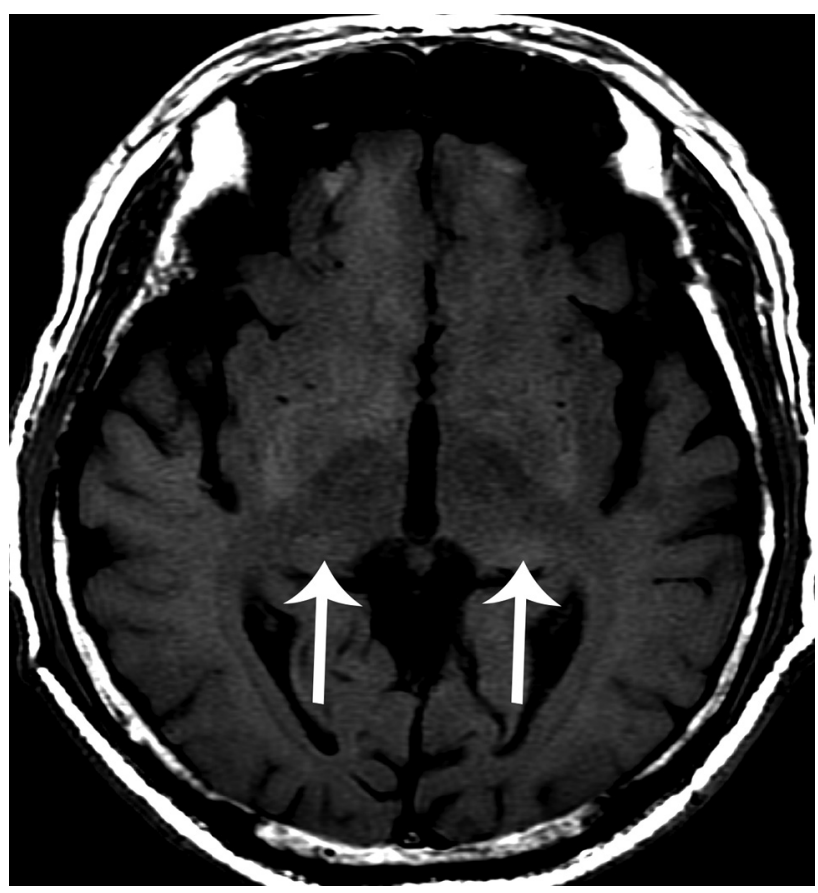

FIG 2. A 63-year-old man with cardiac-type Fabry disease and an IVS4 + 919G > A mutation. A brain MR axial T1-weighted image reveals high signal changes at the bilateral posterior thalamus (arrows), the "pulvinar sign."

system: 0 , absent; 1 , punctate foci; 2 , beginning confluence; and 3, large, confluent.

The degree of elongation and tortuosity of the BA was semiquantitatively evaluated by using the criteria of Smoker et $\mathrm{al}^{9} \mathrm{ac}-$ cording to the height of the bifurcation of the BA and its most lateral position. The scale used to categorize the height of the bifurcation of the BA was as follows: 0 , at or below the dorsum sellae; 1 , within the suprasellar cistern; 2 , at the level of the floor of the third ventricle; and 3, indenting and elevating the floor of the third ventricle. Finally, throughout its course, the most lateral position of the BA was graded as 0 for midline, 1 for the medialto-lateral margins of the clivus or dorsum sellae, 2 for the lateralto-lateral margins of the clivus or dorsum sellae, and 3 for a position in the cerebellopontine angle cistern.

\section{Quantitative Evaluation}

The diameter of the BA was measured on a workstation (AZE Virtual Place Plus; AZE, Tokyo, Japan). An observer blinded to the clinical information of each case drew a line perpendicular to the middle portion of the BA on a sagittal view of the maximumintensity-projections of a 3D time-of-flight MR angiogram (Fig $1 B)$. The observer then recorded the full width at half maximum as the diameter. Vascular stenosis was identified when the BA was determined to be $<2 \mathrm{~mm}$ in diameter.

WMHs were quantitatively calculated by using methods previously described in the literature. ${ }^{10}$ To optimize the accuracy of the WMH registration procedure in a voxelwise analysis scheme, we combined the Diffeomorphic Anatomical Registration Through Exponentiated Lie Algebra-based T1 voxel-based morphometry approach ${ }^{11}$ by using the Gaser voxel-based morphometry 8 toolbox (http://www.neuro.uni-jena.de/vbm/download/) 
Table 1: The brain MR imaging findings of patients with IVS4-type Fabry disease and healthy controls

\begin{tabular}{|c|c|c|c|}
\hline & $\begin{array}{l}\text { IVS4 Fabry } \\
\text { Disease }\end{array}$ & Healthy Control & $P$ Value $^{\mathrm{a}}$ \\
\hline No. & 26 & 26 & \\
\hline Mean age (SD) (years) & $59.3(7.2)$ & $58.4(6.48)$ & .680 \\
\hline $\operatorname{Sex}(M / F)$ & $20: 6$ & $20: 6$ & 1.000 \\
\hline Infarction & $9(35 \%)$ & $0(0 \%)$ & .001 \\
\hline $\begin{array}{l}\text { Area of infarction (anterior circulation/ } \\
\text { posterior circulation/both) }\end{array}$ & $3 / 2 / 4$ & $0 / 0 / 0$ & .012 \\
\hline Hemorrhage/microbleeds & $0(0 \%)$ & $0(0 \%)$ & 1.000 \\
\hline White matter scores ${ }^{\mathrm{b}}$ & $15 / 8 / 2 / 1$ & $19 / 6 / 1 / 0$ & .554 \\
\hline Pulvinar sign & $8(30 \%)$ & $0(0 \%)$ & .002 \\
\hline Chiari I malformation & $0(0 \%)$ & $0(0 \%)$ & 1.000 \\
\hline Vascular stenosis ${ }^{c}$ & $2(8 \%)$ & $2(8 \%)$ & 1.000 \\
\hline Mean diameter of BA (SD) (mm) & $2.75(0.62)$ & $2.47(0.66)$ & .245 \\
\hline Laterality of $\mathrm{BA}^{\mathrm{b}}$ & $12 / 13 / 1 / 0$ & $6 / 18 / 1 / 1$ & .283 \\
\hline Height of BA bifurcation ${ }^{\mathrm{b}}$ & $6 / 10 / 10$ & $9 / 11 / 6$ & .436 \\
\hline
\end{tabular}

isons of BA diameters between groups were evaluated by using a nonparametric Kruskal-Wallis test.

For the analysis of quantitative WMHs, which was associated with total intracranial volume, we normalized each $\mathrm{WMH}$ volume by this variable to rule out an effect of brain size in our statistical models. Three ANCOVAs (periventricular white matter hyperintensity, deep white matter hyperintensity, and total WMH volume) were applied to show group differences in normalized WMH volumes, and we controlled for age as a nuisance variable; the significance level was corrected for multiple comparison issue by using the Bonferroni correction $(P=.016)$. The other threshold for

with the lesion-segmentation toolbox, ${ }^{12}$ which was implemented in Statistical Parametric Mapping (SPM8; http://www.fil.ion. ucl.ac.uk/spm/software/spm8). First, all T1- and T2-weighted images were imported into the lesion-segmentation toolbox with default settings to generate WMH probability maps and binary maps in individual space. Second, all T1-weighted MR images were corrected for bias-field inhomogeneities and were affineregistered to tissue probability maps in the Montreal Neurological Institute standard space (http://www.mni.mcgill.ca/) after tissue segmentation with the same generative model. ${ }^{13}$ These affined tissue segments were iteratively registered to the group-based template, which was generated from all images included in the current study through nonlinear deformation by using the Diffeomorphic Anatomical Registration Through Exponentiated Lie Algebra. Third, all resulting WMH maps (probability and binary) in the individual space were then normalized and modulated by applying nonlinear deformations to compare actual WMH volumetric measurements in the Montreal Neurological Institute standard space.

We divided the whole brain into 2 traditional subtypes according to their location: periventricular WMHs, defined as a $13-\mathrm{mm}$ lining from the ventricle surface, or deep WMHs, which comprised the remaining white matter. This brain atlas with the 2 subtypes was subsequently applied to $\mathrm{WMH}$ volume maps to automatically localize the WMH loci in the Montreal Neurological Institute standard space. Global and regional WMH volumes in various regions were subsequently extracted and calculated. To further control the effect of differences in brain size on global and regional WMH volumes, we used total intracranial volume for each participant to normalize WMH volume information into a whole-brain ratio. ${ }^{10}$

\section{Statistical Analysis}

Statistical analysis was performed by using the Statistical Package for Social Sciences software package (SPSS 18 for Windows; IBM, Armonk, New York). Semi-quantitative evaluations and BA diameters of patients with late-onset Fabry disease were compared with those of 26 healthy controls. Differences in brain MR imaging findings were analyzed by $\chi^{2}$ and Fisher exact tests. Compar- statistical significance was at the $P<.05$ level.

\section{RESULTS \\ Fabry Disease versus Healthy Controls}

Twenty-six patients with Fabry disease and IVS4 + 919G > A mutations, and 26 sex- and age-matched healthy controls underwent MR imaging (Table 1).

The neurologic symptoms in the group with IVS4-type Fabry vertigo $(n=3,12 \%)$, sudden onset of numbness or weakness $(n=3,12 \%)$, depression $(n=2,8 \%)$, asymmetric facial expression $(n=1,4 \%)$, blurred vision or diplopia $(n=1,4 \%)$, and personality changes $(n=1,4 \%)$. Fourteen patients had symptoms, while the other 12 patients were free of neurologic symptoms. No significant correlation was found between neurologic manifestations and MR imaging findings.

Infarctions were found in 9 patients with IVS4-type Fabry disease (35\%), including 6 men and 3 women, and no infarctions were identified in healthy controls $(P=.001)$. All were lacunar infarctions involving the anterior and/or posterior circulation variably. A 54-year-old man had symptomatic lacunar infarctions, and the other 8 patients were asymptomatic.

Positive pulvinar signs were found in 8 patients with Fabry disease $(30 \%)$ and in no individual in the healthy control group $(0 \%)(P=.002)$. In terms of WMHs, no significant difference was found in the Fazekas scores in all groups; however, quantitative deep WMH volume was significantly higher in patients with IVS4-type Fabry disease $\left(1.1583 \mathrm{~cm}^{3}\right)$ than in individuals in the healthy control data base $\left(0.1354 \mathrm{~cm}^{3}\right)(P=$ .004) (Fig 3).

Mean BA diameter was $2.75 \pm 0.62 \mathrm{~mm}$ in the IVS4-type Fabry disease and $2.47 \pm 0.66 \mathrm{~mm}$ in the healthy control group $(P=.245)$. A 63-year-old male patient with Fabry disease was excluded from further analysis due to dissection of the BA. There was no significant difference in the height of the BA bifurcation $(P=.436)$ and the laterality of the BA $(P=.283)$ between those with IVS4-type Fabry disease and healthy controls. disease were forgetfulness $(n=6,24 \%)$, tinnitus $(n=4,16 \%)$, 


\section{Infarction versus Noninfarction Groups}

Comparisons of brain MR imaging findings in patients with the IVS4-type Fabry disease revealed that patients with infarctions were older (age range, 54-71 years; median age, 65 years) than patients without infarctions (age range, 43-64 years; median age, 58 years $)(P=.009)$ (Table 2$)$. In the infarction group, the Fazekas scale scores for deep white matter hyperintensities were also higher than those of noninfarction group $(P=.023)$. Moreover, 2 and no patients had vascular stenosis in the infarction and noninfarction groups, respectively $(P=.041)$. There was no significant difference in the mean BA diameter between the infarction and noninfarction groups $(P=.301)$.

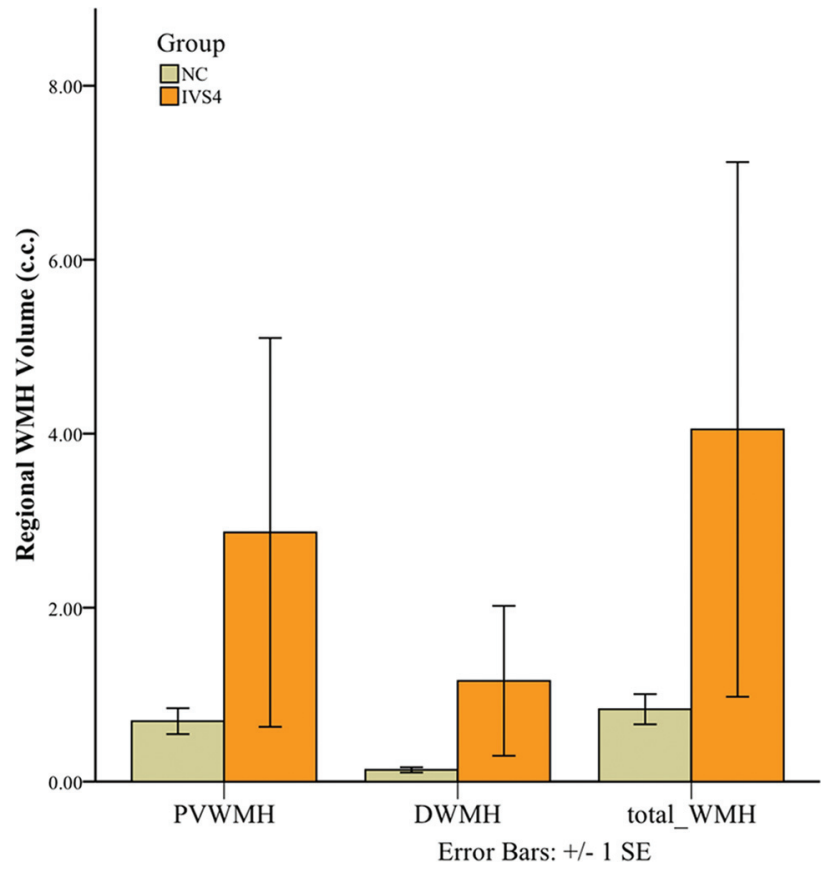

FIG 3. Bar graph of the averaged volume of regional white matter hyperintensities at the periventricular white matter (PVWMH), deep white matter (DWMH), and total white matter in both patients with IVS4-type Fabry disease and healthy controls.

Table 2: Comparison of brain MR imaging findings in patients with IVS4-type Fabry disease, with and without brain infarctions

\begin{tabular}{|c|c|c|c|}
\hline & Infarction & Noninfarction & $P$ Value $^{\mathrm{a}}$ \\
\hline No. & 9 & 17 & \\
\hline Mean age (SD) (years) & $64.0(5.2)$ & $56.8(7.0)$ & .009 \\
\hline $\operatorname{Sex}(M / F)$ & $6: 3$ & $14: 3$ & .332 \\
\hline $\begin{array}{l}\text { Area of infarction (anterior circulation/ } \\
\text { posterior circulation/both) }\end{array}$ & $3 / 2 / 4$ & $0 / 0 / 0$ & .012 \\
\hline Hemorrhage/microbleeds & $0(0 \%)$ & $0(0 \%)$ & 1.000 \\
\hline White matter scores ${ }^{\mathrm{b}}$ & $2 / 4 / 2 / 1$ & $13 / 4 / 0 / 0$ & .023 \\
\hline Pulvinar sign & $3(33 \%)$ & $5(29 \%)$ & .837 \\
\hline Chiari I malformation & $0(0 \%)$ & $0(0 \%)$ & 1.000 \\
\hline Vascular stenosis $^{\mathrm{c}}$ & $2(22 \%)$ & $0(0 \%)$ & .041 \\
\hline Mean diameter of BA (SD) (mm) & $2.57(0.64)$ & $2.86(0.61)$ & .301 \\
\hline Laterality of $\mathrm{BA}^{\mathrm{b}}$ & $4 / 5 / 0 / 0$ & $8 / 8 / 1 / 0$ & .732 \\
\hline Height of BA bifurcation ${ }^{\mathrm{b}}$ & $3 / 5 / 1$ & $3 / 5 / 9$ & .114 \\
\hline
\end{tabular}

\footnotetext{
a $P$, unadjusted.

b Scale of $0 / 1 / 2 / 3$

c Presence of focal stenosis of all intracranial arteries.
}

\section{Sex-Based Differences between Patients with IVS4 Fabry Disease versus Healthy Controls}

There was no difference in brain MR imaging findings between female patients having Fabry disease and healthy controls (Table 3). However, in male patients with Fabry disease (Table 4), we observed a higher incidence of infarctions $(P=.010)$ and pulvinar signs $(P=.004)$ and larger BA diameters $(2.91 \pm 0.60 \mathrm{~mm}, P=$ $.041)$ compared with healthy controls. A receiver operating characteristic curve analysis of the BA diameter in male patients revealed that a threshold of $2.95 \mathrm{~mm}$ could achieve a sensitivity of $53 \%$ and a specificity of $85 \%$ in differentiating IVS4-type Fabry disease and healthy controls (area under the curve $=0.69 ; 95 \%$ confidence interval, 0.52-0.86).

\section{DISCUSSION}

In classic Fabry disease, stroke is a common and important manifestation. ${ }^{5-16}$ It could be a consequence of cardiogenic embolism from cardiomyopathy, valvular heart disease, ischemic heart disease, and/or arrhythmias. Besides, due to the deposition of neutral glycosphingolipids in vascular endothelial cells, the potential cerebrovascular damage or alterations in brain perfusion should be considered. ${ }^{17}$ The prevalence of stroke in patients with IVS4type Fabry disease was similar to that reported by a previous retrospective study of classic Fabry disease. ${ }^{15}$ However, the median age of our patients having IVS4-type Fabry disease with infarctions was older than the prior reported median age, which ranged from 28 to 54 years. ${ }^{18}$ Furthermore, none of the young patients with IVS4-type Fabry disease (younger than 54 years of age) in our cohort had experienced stroke or asymptomatic infarctions, even though Fabry disease poses a higher relative risk of stroke across all age groups. ${ }^{14}$ In our cohort, the mean age of patients with infarctions was older than that in patients without infarctions. This study demonstrated that Fabry disease with IVS4 + 919G $>$ A mutations, though previously categorized as a cardiac variant, also increased the risk of stroke in a late-onset fashion.

Multiple or confluent WMHs on T2-weighted images are one of the characteristic neuroradiologic signs of classic Fabry disease. The injury is caused by globotriaosylceramide (GL-3)-related endothelial damage and subsequent microvascular degeneration. ${ }^{19}$ Although we observed no significant difference in WMH severity between the 2 groups by using the semi-quantitative Fazekas score, the quantitative deep WMH volumes of IVS4-type Fabry disease were significantly higher than those in healthy controls; this finding was consistent with the higher sensitivity of the automated segmentation method for WMHs. ${ }^{20}$

Vertebrobasilar dolichoectasia is thought to be an early and frequent sign of classic Fabry disease. ${ }^{21}$ For example, Fellgiebel et $\mathrm{al}^{22,23}$ demonstrated that the BA diameter was superior to white matter lesions as a diagnostic tool of Fabry disease and that this sign could be used for screening in young patients who have experienced stroke. More specifically, their group used a diameter of $2.98 \mathrm{~mm}$ as a cutoff value with a sensi- 
Table 3: Brain MR imaging findings of female patients with IVS4-type Fabry disease and healthy controls

\begin{tabular}{|c|c|c|c|}
\hline & $\begin{array}{c}\text { Late-Onset } \\
\text { Fabry Disease }\end{array}$ & Healthy Control & $P$ Value $^{\mathrm{a}}$ \\
\hline No. & 6 & 6 & \\
\hline Mean age (SD) (years) & $57.8(6.5)$ & $58.0(6.2)$ & .937 \\
\hline Infarction & $3(50 \%)$ & $0(0 \%)$ & .091 \\
\hline $\begin{array}{l}\text { Area of infarction (anterior circulation/ } \\
\text { posterior circulation/both) }\end{array}$ & $2 / 1 / 0$ & $0 / 0 / 0$ & .135 \\
\hline Hemorrhage/microbleeds & $0(0 \%)$ & $0(0 \%)$ & 1.000 \\
\hline White matter scores ${ }^{\mathrm{b}}$ & $2 / 3 / 1 / 0$ & $5 / 1 / 0 / 0$ & .193 \\
\hline Pulvinar sign & $1(17 \%)$ & $0(0 \%)$ & .500 \\
\hline Chiari I malformation & $0(0 \%)$ & $0(0 \%)$ & 1.000 \\
\hline Vascular stenosis $^{c}$ & $1(17 \%)$ & $0(0 \%)$ & .500 \\
\hline Mean diameter of BA (SD) (mm) & $2.25(0.41)$ & $2.62(0.42)$ & .180 \\
\hline Laterality of $\mathrm{BA}^{\mathrm{b}}$ & $1 / 5 / 0 / 0$ & $1 / 4 / 0 / 1$ & .574 \\
\hline Height of BA bifurcation ${ }^{\mathrm{b}}$ & $2 / 2 / 2$ & $2 / 1 / 3$ & .766 \\
\hline
\end{tabular}

Table 4: Brain MR imaging findings of male patients with IVS4 +919G > A and healthy controls

\begin{tabular}{|c|c|c|c|}
\hline & $\begin{array}{l}\text { Late-Onset } \\
\text { Fabry Disease }\end{array}$ & Healthy Control & $P$ Value \\
\hline No. & 20 & 20 & \\
\hline Mean age (SD) (years) & $59.7(7.5)$ & $58.5(6.7)$ & .547 \\
\hline Infarction & $6(30 \%)$ & $0(0 \%)$ & .010 \\
\hline $\begin{array}{l}\text { Area of infarction (anterior circulation/ } \\
\text { posterior circulation/both) }\end{array}$ & $1 / 1 / 4$ & $0 / 0 / 0$ & .070 \\
\hline Hemorrhage/microbleeds & $0(0 \%)$ & $0(0 \%)$ & 1.000 \\
\hline White matter scores ${ }^{\mathrm{b}}$ & 13/5/1/1 & $14 / 5 / 1 / 0$ & .792 \\
\hline Pulvinar sign & $7(35 \%)$ & $0(0 \%)$ & .004 \\
\hline Chiari I malformation & $0(0 \%)$ & $0(0 \%)$ & 1.000 \\
\hline Vascular stenosis $^{c}$ & $1(5 \%)$ & $2(10 \%)$ & .513 \\
\hline Mean diameter of BA (SD) (mm) & $2.91(0.60)$ & $2.43(0.72)$ & .041 \\
\hline Laterality of $\mathrm{BA}^{\mathrm{b}}$ & $11 / 8 / 1 / 0$ & $5 / 14 / 1 / 0$ & .143 \\
\hline Height of BA bifurcation ${ }^{\mathrm{b}}$ & $4 / 8 / 8$ & $7 / 10 / 3$ & .191 \\
\hline
\end{tabular}

\footnotetext{
${ }^{a} P$, unadjusted.

${ }^{b}$ Scale of $0 / 1 / 2 / 3$.

c Presence of focal stenosis of all intracranial arteries.
}

tivity of $84 \%$ and a specificity of $88.5 \%$. Furthermore, Uçeyler et $\mathrm{al}^{24}$ proposed that a BA diameter of $>3.2 \mathrm{~mm}$ could distinguish a male patient with Fabry disease and healthy controls with a sensitivity of $87 \%$ and specificity of $86 \%$. In our cohort, male patients with IVS4 Fabry disease had significantly larger BA diameters than healthy controls, while female patients did not. A cutoff of $3.2 \mathrm{~mm}$ for late-onset Fabry disease had a low sensitivity, while a cutoff of $2.95 \mathrm{~mm}$ had fair sensitivity and specificity. This difference is probably because this was a group with late-onset disease. Thus, long-term follow-up of these patients is needed for a more thorough understanding of the progression and severity of IVS4type Fabry disease. The concept offered an explanation for no significant difference in MR imaging findings of the female group between healthy controls and those with late-onset X-linked disease.

The characteristic "pulvinar sign," which comprises hyperintensities of the posterior thalamus on T1-weighted images, is presumed to occur secondary to increased cerebral blood flow in the posterior circulation. ${ }^{7,25}$ In our patient cohort, we observed this sign in $8(30 \%)$ patients with IVS4-type Fabry disease (Fig 2). This result is consistent with the hypothesis that this mutated form of Fabry disease comprises neurovascular alterations and cardiac and renal involvement.
This study had some limitations. First, IVS4-type Fabry disease has been previously considered a cardiac variant, which the pathologic changes of the brain was not well recognized. This limitation explains the small number of brain MRIs in this retrospective study, though the incidence of this type of Fabry disease is common in our population. An aim of this study was also to demonstrate that involvement of this type is not limited to the heart alone. Second, Fabry disease involves multiple organs, and our patients often had hypertension and cardiac and renal disease. We did not evaluate the effects of these comorbidities on intracranial conditions due to the small number of cases reviewed. Third, despite healthy controls not having cardiac and renal abnormalities, our retrospective study could not exclude the possibility of asymptomatic or undiagnosed Fabry disease in the healthy control group. Fourth, the sample size of patients with IVS4 mutation is small and may not allow detection of the milder and later-onset pathologic changes in female patients as opposed to male patients. A larger sample size and long-term follow-up are crucial for understanding late manifestations of clinical symptoms and imaging findings. Last, the age and sex-matched comparison between IVS4-type and classic Fabry disease was not included in this study due to the rarity of classic Fabry disease.

\section{CONCLUSIONS}

This study demonstrated that the cardiac-type Fabry disease with a mutation of IVS4 $+919 \mathrm{G}>\mathrm{A}$ was not limited to involvement of the cardiac system. Patients with this form of the disease presented with a greater amount of deep white matter intensities and higher incidences of infarctions and pulvinar signs than healthy controls. Finally, the use of a BA cutoff diameter of $2.95 \mathrm{~mm}$ achieved fair sensitivity and specificity in differentiating male patients with IVS4-type Fabry disease and healthy controls. Awareness of the neuroimaging findings helps early detection and treatment of the specific type of Fabry disease.

\section{REFERENCES}

1. Desnick RJ, Ioannou YA, Eng CM. $\boldsymbol{\alpha}$-galactosidase A deficiency: Fabry disease. In: Scriver CR, ed. The Metabolic and Molecular Bases of Inherited Disease. 8th ed. New York: McGraw-Hill; 2001:3733-74

2. Reisin RC, Romero C, Marchesoni C, et al. Brain MRI findings in patients with Fabry disease. J Neurol Scis 2011;305:41-44 CrossRef Medline

3. Chong KW, Lu YH, Hsu JH, et al. High incidence of cardiac variant of Fabry disease in Taiwanese revealed by newborn screening. In: 
Proceedings of the Taiwan Human Genetics Society Autumn Symposium, Hualien, Taiwan. October 4-5, 2008

4. Lin HY, Chong KW, Hsu JH, et al. High incidence of the cardiac variant of Fabry disease revealed by newborn screening in the Taiwan Chinese population. Circ Cardiovasc Genet 2009;2:450-56 CrossRef Medline

5. Chien YH, Lee NC, Chiang SC, et al. Fabry disease: incidence of the common later-onset $\alpha$-galactosidase A IVS4+919G $\rightarrow$ A mutation in Taiwanese newborns: superiority of DNA-based to enzymebased newborn screening for common mutations. Mol Med 2012; 18:780-84 CrossRef Medline

6. Ishii S, Nakao S, Minamikawa-Tachino R, et al. Alternative splicing in the alpha-galactosidase A gene: increased exon inclusion results in the Fabry cardiac phenotype. Am J Hum Genet 2002;70:994-1002 CrossRef Medline

7. Takanashi Ji, Barkovich AJ, Dillon WP, et al. T1 hyperintensity in the pulvinar: key imaging feature for diagnosis of Fabry disease. AJNR Am J Neuroradiol 2003;24:916-21 Medline

8. Fazekas F, Chawluk JB, Alavi A, et al. MR signal abnormalities at $\mathbf{1 . 5}$ $\mathrm{T}$ in Alzheimer's dementia and normal aging. AJR Am J Roentgenol 1987;149:351-66 CrossRef Medline

9. Smoker WR, Corbett JJ, Gentry LR, et al. High-resolution computed tomography of the basilar artery, 2: vertebrobasilar dolichoectasia-clinical-pathologic correlation and review. AJNR Am J Neuroradiol 1986;7:61-72 Medline

10. Tsai A, Huang CC, Yang AC, et al. Association of BACE1 gene polymorphism with cerebellar volume but not cognitive function in normal individuals. Dement Geriatr Cogn Dis Extra 2012;2:632-37 CrossRef Medline

11. Ashburner J. A fast diffeomorphic image registration algorithm. Neuroimage 2007;38:95-113 CrossRef Medline

12. Schmidt P, Gaser C, Arsic M, et al. An automated tool for detection of FLAIR-hyperintense white-matter lesions in multiple sclerosis. Neuroimage 2012;59:3774-83 CrossRef Medline

13. Ashburner J, Friston KJ. Unified segmentation. Neuroimage 2005;26: 839-51 CrossRef Medline

14. Sims K, Politei J, Banikazemi M, et al. Stroke in Fabry disease frequently occurs before diagnosis and in the absence of other clinical events: natural history data from the Fabry registry. Stroke 2009;40: 788-94 CrossRef Medline

15. Buechner S, Moretti M, Burlina AP, et al. Central nervous system involvement in Anderson-Fabry disease: a clinical and MRI retrospective study. J Neurol Neurosurg Psychiatry 2008;79:1249-54 CrossRef Medline

16. Rolfs A, Böttcher T, Zschiesche M, et al. Prevalence of Fabry disease in patients with cryptogenic stroke: a prospective study. Lancet 2005;366:1794-96 CrossRef Medline

17. Fellgiebel A, Müller MJ, Ginsberg L. CNS manifestations of Fabry's disease. Lancet Neurol 2006;5:791-95 CrossRef Medline

18. Kolodny E, Fellgiebel A, Hilz MJ, et al. Cerebrovascular involvement in Fabry disease: current status of knowledge. Stroke 2015;46: 302-13 CrossRef Medline

19. deVeber GA, Schwarting GA, Kolodny EH, et al. Fabry disease: immunocytochemical characterization of neuronal involvement. Ann Neurol 1992;31:409-15 CrossRef Medline

20. Prins ND, van Straaten EC, van Dijk EJ, et al. Measuring progression of cerebral white matter lesions on MRI: visual rating and volumetrics. Neurology 2004;62:1533-39 CrossRef

21. Politei J, Schenone AB, Burlina A, et al. Vertebrobasilar dolichoectasia in Fabry disease: the earliest marker of neurovascular involvement? JIEMS 2014;2:2326409814541246 CrossRef

22. Fellgiebel A, Keller I, Marin D, et al. Diagnostic utility of different MRI and MR angiography measures in Fabry disease. Neurology 2009;72:63-68 CrossRef Medline

23. Fellgiebel A, Keller I, Martus P, et al. Basilar artery diameter is a potential screening tool for Fabry disease in young stroke patients. Cerebrovasc Dis 2011;31:294-99 CrossRef Medline

24. Uçeyler N, Homola GA, Guerrero González H, et al. Increased arterial diameters in the posterior cerebral circulation in men with Fabry disease. PLoS One 2014;9:e87054 CrossRef Medline

25. Moore DF, Ye F, Schiffmann R, et al. Increased signal intensity in the pulvinar on T1-weighted images: a pathognomonic MR imaging sign of Fabry disease. AJNR Am J Neuroradiol 2003;24:1096-101 Medline 